\title{
Electrocardiographic and Echocardiographic Changes in Subarachnoid Hemorrhage and Their Final Impact on Early Outcome: A Prospective Study Before and After the Treatment
}

\author{
Shokoufeh Hajsadeghi ${ }^{\mathrm{a}}$, Reza Mollahoseini ${ }^{\mathrm{b}}$, Babak Alijani ${ }^{\mathrm{c}}$, Nazli Sadeghid ${ }^{\mathrm{d}}$, Mohammad Javad Manteghie, \\ Mohammad Hossein Lashkarif , Morteza Hassanzadeh ${ }^{\mathrm{g}}$, h
}

\begin{abstract}
Background: We aimed to prospectively investigate the changes in the electrocardiography (ECG) and the echocardiography of the patients with subarachnoid hemorrhage $(\mathrm{SAH})$ before and after treatment, and to evaluate the overall role of the findings on early patients' outcome.
\end{abstract}

Methods: All consecutive patients with SAH were evaluated with onadmission ECG and echocardiography. For those with an abnormal result, a second evaluation was performed after the therapeutic interventions. All of the participants were followed until discharged or possibly expired in the hospital. Proper statistical methods were used to compare the changes between the two groups of the patients: the "expired" group, and the "discharged" group.

Results: Of the total of 60 subjects, 25 (41.6\%) and three (5\%) had an abnormal ECG and echocardiography that were dropped to four $(6.7 \%)$ and one $(1.7 \%)$ after treatment, respectively. The most frequent ECG finding was T-wave inversion. Six subjects (10\%) were expired in the hospital. Abnormal primary ECG was found in five out of the six dead subjects (83.3\%) and 20 out of the 54 discharged ones $(37 \%)(\mathrm{P}=0.029)$. None of the three patients with abnormal primary echocardiograms were expired during the hospitalization.

Conclusion: Most SAH-induced changes in the ECG and the echocardiography are transient and reversible. Abnormal ECG is a good

\footnotetext{
Manuscript accepted for publication December 22, 2014

aDepartment of Cardiology, Rasoul-e-Akram Hospital, Iran University of Medical Sciences, Tehran, Iran

bepartment of Neurosurgery, Firoozgar Hospital, Iran University of Medical Sciences, Tehran, Iran

'Department of Neurosurgery, Poursina Hospital, Guilan University of Medical Sciences, Rasht, Iran

dShahid Beheshti University of Medical Sciences, Tehran, Iran

'Faculty of Medicine, Iran University of Medical Sciences, Tehran, Iran

fDepartment of Surgery, AJA University of Medical Sciences, Tehran, Iran

gDepartment of Internal Medicine, Rasoul-e-Akram Hospital, Iran University of Medical Sciences, Tehran, Iran

${ }^{\mathrm{h}}$ Corresponding Author: Morteza Hassanzadeh, Rasoul-e-Akram Medical Center, Niyayesh Street, Sattarkhan Ave., Tehran, Iran.

Email: Morteza_hb@yahoo.com
}

doi: http://dx.doi.org/10.14740/jnr317w predictor of inpatient mortality, but abnormal echocardiography is not.

Keywords: Subarachnoid hemorrhage; Electrocardiography; Echocardiography; Outcome

\section{Introduction}

Subarachnoid hemorrhage (SAH), as a catastrophic neurologic event, usually affects middle-aged population with a rather greater incidence in women [1]. The most common causes of SAH include head trauma and rupture of a saccular aneurysm. Bleeding from an arteriovenous malformation, extension of a primary intracerebral hemorrhage into the subarachnoid space, and idiopathic SAH are among other etiologies. After the primary brain damage, the early post-SAH mortality rate has been reported ranging from $30 \%$ to $45 \%$ in different studies [2-4]. In addition, some other injuries may also occur from minutes to weeks after the initial event [4] that influence the patients' outcome.

Neurological complications including cerebral vasospasm and rebleeding are frequently considered as the main causes of SAH-related mortality. Nevertheless, non-neurological complications of SAH such as hematologic alterations $[5,6]$, electrolyte disturbances [7,8], and cardiac abnormalities are both common and potentially life-threatening [9]. Among all, cardiac manifestations after SAH have been well described for many years $[10,11]$; different types of abnormalities on electrocardiogram (ECG) and also some echocardiographic changes in the function of left ventricule (LV) have been reported. The final impact of cardiac findings on prognosis, however, has not been well understood. Moreover, prospective studies on the LV function before and after SAH treatment have been less described.

We designed this study, therefore, to prospectively investigate the ECG patterns of cardiac injury along with the echocardiographic patterns of segmental LV dysfunction in patients with SAH before and after therapeutic interventions, and to evaluate the overall role of the findings on early patients' outcome. 


\section{Materials and Methods}

\section{Patient selection}

Study subjects were recruited from SAH patients admitted to the Rasoul-e-Akram General Hospital, Tehran, Iran. During 1 -year course of the study, all consecutive patients aged $>20$ years with confirmed diagnosis of SAH by computed tomography (CT) scan of the brain were recruited. Excluded were patients with any sign of intracerebral hemorrhage, subdural hemorrhage or epidural hemorrhage on the brain CT scan as well as those with any history of myocardial infarction, cardiomyopathy, congestive heart failure or valvular heart disease.

\section{Study protocol}

After enrollment, demographic and clinical data were collected from the patients, their family members and the available medical records. Before therapeutic interventions, a standard 12-lead ECG and a transthoracic echocardiography were performed for all patients and any abnormalities were recorded. All ECG abnormalities were confirmed by a senior cardiologist among the authors ( $\mathrm{SH}$ ) who performed all echocardiographic evaluations as well. For those patients with an abnormal initial ECG or echocardiography, a second evaluation was planned after the medical and/or surgical interventions. All of the subjects were followed until discharged or possibly expired in the hospital.

The study protocol was approved by the ethics committee of the Iran University of Medical Sciences and a signed consent form was obtained from all of the participants.

\section{Study variables}

Baseline characteristics included patients' age and sex, and history of risk factors such as hypertension and smoking. The etiology of SAH was classified as traumatic SAH, SAH due to a ruptured aneurysm (assessed by cerebral angiography), and SAH of unknown origin (despite assessment by cerebral angiography).

For echocardiographic evaluation, the following images were obtained: parasternal long axis, parasternal short axis (at the level of the mitral valve, papillary muscles and apex), apical two-, three-, and four-chamber views, subcostal long axis and subcostal short axis (at the level of the mitral valve, papillary muscles and apex). LV ejection fraction (LVEF), LV size (end-diastolic and end-systolic diameters), and ventricular wall motion abnormalities (WMAs) were also measured (including the septum and the LV posterior wall).

\section{Statistical analyses}

Statistical analyses were performed using the software SPSS version 20.0. Descriptive statistics were performed, and results
Table 1. Demographic and Clinical Characteristics of the Study Patients

\begin{tabular}{ll}
\hline $\begin{array}{l}\text { Age (years)* } \\
\text { Sex** }\end{array}$ & $52 \pm 15$ \\
Male & $28(47)$ \\
Female & $32(53)$ \\
Risk factors** & \\
Hypertension & $15(25)$ \\
$\quad$ Smoking & $11(18.3)$ \\
Etiology of subarachnoid hemorrhage** & \\
$\quad$ Aneurysm & $36(60)$ \\
$\quad$ Trauma & $6(10)$ \\
Idiopathic & $18(30)$ \\
Duration of hospitalization (days)* & $23 \pm 16$ \\
Outcome** & \\
Discharged & $54(90)$ \\
Expired & $6(10)$ \\
\hline
\end{tabular}

*Values are the mean \pm standard deviation. ${ }^{*}$ Values are the number (percentage).

were expressed as proportions and mean \pm standard deviation (SD). The Student's $t$-test, the Mann-Whitney U test, the Fisher's exact test, or the Chi-square test (and/or the correlation coefficient) were used for between-group comparisons where appropriate. All tests were two-tailed and $\mathrm{P}<0.05$ was considered as statistically significant.

\section{Results}

\section{Baseline characteristics}

A total number of 60 subjects were recruited to this study, 32 $(53 \%)$ of whom were females. The mean \pm SD of age of the participants was $52 \pm 15$ years. In 18 subjects (30\%) the etiology of SAH was unknown, $36(60 \%)$ had ruptured aneurysm, and six $(10 \%)$ had traumatic SAH.

The mean duration of hospitalization was 23 days (SD $16)$, and six subjects (10\%) were expired in the hospital. Table 1 summarizes the baseline characteristics of the study participants.

\section{Results of the cardiac evaluations}

The result of primary ECG was normal for 35 subjects (58.4\%). Of the other 25 subjects, 14 (23.3\% of total SAH samples) had $\mathrm{T}$ inversion, five $(8.3 \%)$ had $\mathrm{T}$ inversion and ST depression, two $(3.3 \%)$ had T inversion and ST elevation, two (3.3\%) had QT prolongation and ST depression, one (1.7\%) had ST elevation, and one (1.7\%) had QT prolongation (Table 2$)$. The second ECG, after therapeutic interventions, demonstrated persis- 
Table 2. Results of Cardiac Evaluations of the Study Subjects Before Therapeutic Interventions

\begin{tabular}{|c|c|}
\hline \multicolumn{2}{|l|}{ Electrocardiography (ECG) } \\
\hline Normal ECG* ${ }^{*}$ & $35(58.4)$ \\
\hline $\mathrm{T}$ inversion* & $21(35)$ \\
\hline ST depression* & $7(11.7)$ \\
\hline ST elevation* & $3(5)$ \\
\hline QT prolongation* & $3(5)$ \\
\hline \multicolumn{2}{|l|}{ Echocardiography } \\
\hline Normal* & $57(95)$ \\
\hline Wall motion abnormality* & $3(5)$ \\
\hline Left ventricular (LV) ejection fraction $(\%)^{* *}$ & $62 \pm 6$ \\
\hline LV-end systolic dimension $(\mathrm{cm})^{* *}$ & $3.2 \pm 1.2$ \\
\hline LV-end diastolic dimension $(\mathrm{cm})^{* *}$ & $4.6 \pm 0.5$ \\
\hline
\end{tabular}

*Values are the number (percentage of the total sample). ${ }^{*}$ Values are the mean \pm standard deviation.

tent $\mathrm{T}$ inversion in three ( $5 \%$ of total $\mathrm{SAH}$ samples), persistent ST elevation in one $(1.7 \%)$, and normal results in the other 21 (35\%).

On the primary echocardiography, the mean \pm SD of LVEF was $62 \pm 6 \%$ for all participants, and three $(5 \%)$ of the subjects had WMA in the initial evaluation. Other results are shown in the Table 2.

After treatment, on the second echocardiographic evaluation, one subject had persistent WMA, whereas results were normal for the two others. The first case had LVEF of $46 \%$ with apical dyskinesia and anterior wall akinesia on admission that returned to the normal after the treatment $(\mathrm{LVEF}=65 \%$ and normal wall motion). The primary LVEF for the second case was $50 \%$ along with the base of septum and anterior septal wall hypokinesis; both became normal after the treatment (LVEF $=63 \%$ and normal wall motion). The third case, however, had LVEF of $50 \%$ with the inferior wall akinesia and mild septal hypokinesis on admission which were persistent after the treatment. None of the three patients with abnormal primary echocardiograms were expired during the hospitalization.

\section{Role of the findings on the patients' outcome}

After statistical adjustment by multivariate analysis, primary ECG was highly predictive of the patient's outcome (Table 3): abnormal primary ECG was found in five out of the six dead subjects $(83.3 \%)$ and 20 out of the 54 discharged ones $(37 \%)$ $(\mathrm{P}=0.029)$. The mean $\pm \mathrm{SD}$ for the duration of hospitalization of the subjects with normal ECG (21 \pm 11 days) was lesser than those with abnormal ECG ( $25 \pm 12$ days), but this difference was not statistically significant $(\mathrm{P}=0.601)$.

The mean LVEF was $62 \%$ in the discharged group (SD $6.4 \%$ ) and $60 \%$ in the expired group (SD 2.9\%) which showed no significant statistical difference $(\mathrm{P}=0.527)$.

There was no correlation between the duration of hospitalization, the in-patient mortality rate, the LV size (LV end systole and LV end diastole) or the abnormal echocardiography (WMA).

\section{Discussion}

In the present study, 25 out of 60 enrolled patients $(41.6 \%)$ with SAH had an abnormal on-admission ECG, among whom 21 returned to normal after treatment. T-wave inversion was the most frequent ECG finding both at presentation (35\%) and post-treatment $(5 \%)$. There was a good correlation between having an abnormal ECG and poor outcome $(\mathrm{P}=0.029)$. We also found three cases (5\%) with WMA on echocardiography, two of whom became normal after SAH treatment. The echocardiographic findings, however, had no correlation with outcome.

Cardiac manifestations of SAH, including abnormalities on ECG and echocardiography, are a well-accepted phenomenon today. After the first description by Byer et al [12] in 1947, ECG changes in patients with SAH have been frequently investigated. The reported prevalence of ECG abnormalities varies from $27 \%$ to $100 \%$ among SAH patients [13-15], with rate of reversion to normal ranging from $40 \%$ to $100 \%$ in different studies [16, 17]. Such factors as differences in the baseline ECG of the patients before SAH, methods of ECG evaluation, or the study designation (especially in terms of timing and frequency of ECG recordings) might have been involved in creating these varying results. According to Harries et al [18], ECG abnormalities may occur as late as 2 weeks after the onset of SAH, and may persist up to 6 weeks or even, as noted elsewhere [17], up to some months. Keeping those points in mind, our findings next to the others' are best described when considering SAH-related ECG changes as a continuum, with early-initiating and/or early-reverting changes at one end and delayed-initiating or delayed-reverting ones at the other end of the continuum.

Although some investigators, similar to us, found T-wave inversion as the most common post-SAH ECG abnormality $[17,19]$, others reported something else $[11,13,18,20]$. Near-

Table 3. Role of the Electrocardiographic and Echocardiographic Results on the Patients' Outcome

\begin{tabular}{llll}
\hline & Discharged $(\mathbf{N}=\mathbf{5 4})$ & Expired $(\mathbf{N}=\mathbf{6})$ & $\mathbf{P}$ \\
\hline Abnormal electrocardiogram* & $20(37)$ & $5(83.3)$ & 0.029 \\
Wall motion abnormality* & $3(5.6)$ & $0(0)$ & 0.238 \\
Left ventricular ejection fraction $(\%) * *$ & $62 \pm 6$ & $60 \pm 3$ & 0.527 \\
\hline
\end{tabular}

*Values are the number (percentage in the group). ${ }^{* *}$ Values are the mean \pm standard deviation. 
ly all of the commonly reported ECG changes, however, have been included the repolarization waveforms (ST segment, T and $U$ waves), which reflects a subendocardial damage and reinforces the "catecholamine storm" theory $[14,17,21]$ as the responsible mechanism for SAH-related cardiac involvements: rapidly excessive catecholamine release after SAH results in subendocardial contraction band necrosis. The overall influence of the ECG changes on patients' outcome has also been a matter of dispute, whereas the observations by many authors, in support of our findings, are in favor of ECG playing a role in the outcome $[11,22]$; others found no prognostic value for ECG abnormalities [14, 17, 23-25]. The same viewpoint, mentioned earlier, can also explain different results on both the types of the ECG changes after SAH and their presumed effect on patients' outcome. The continuum here, then, is theoretized to be from minimal nonspecific ST-T changes with no clinical significance to severe ECG abnormalities of high impact on prognosis, an issue which has also been addressed by Samuels in 1987 [26].

Echocardiographic evidences of LV dysfunction after $\mathrm{SAH}$ have been reported in a wide incidence range (from 3\% to $60 \%$ ) by many authors $[24,27-30]$. Some also found $25-$ $100 \%$ reversibility of echocardiographic changes by time $[25$, 31]. In some studies [31-33], similar to this investigation, LV dysfunction on echocardiography was considered as not having a role to predict patients' outcome, while it was proved as a predictor of poor outcome in some others [34-36]. Taking into account different study definitions (in terms of abnormal echocardiogram in the context of SAH) and designs (prospective vs. retrospective), and different times of the follow-up echocardiographies, those varying results could be better understood. In this study, the WMA in one of the three patients remained unchanged after treatment. Probably either it would also be corrected if a more delayed echocardiography had been performed, or that was a background cardiac problem before the onset of SAH. Zaroff et al [33] demonstrated that the regional WMA seen in the SAH patients does not match the territory of a specific coronary artery. Among all the occurred patterns of WMA, they found inferior wall pattern as the least common. Of note, the inferior wall was involved in the single patient with persistent WMA after treatment. That observation might suggest the normalization of the inferior wall involvement after SAH occurs later than other patterns; more studies, however, are needed to address this interesting issue.

This study was conducted in a general (rather than referral) hospital, and all echocardiographies were performed by a single cardiologist. The former feature along with the prospective designation minimized the potential sources of bias compared to the similar studies, and the latter one increased the reliability of our data set.

We enrolled SAH patients of any etiology that made our sample heterogenous. Additionally, the difficulties inherent in prospective investigations limited the number of our enrolled subjects to 60 . Fortunately, despite those limitations, our final patient population had a typical demographic distribution for SAH. In this study the patients were evaluated during hospitalization; our results, therefore, cannot be applied for long term outcome of the SAH patients. Finally, we did not consider the graded severity of SAH in our evaluation. For future studies it is recommended to use the available classification schemes (such as Hunt-Hess scale) to compare the findings among patients with different SAH grades.

In summary, we prospectively investigated the ECG and echocardiography of $60 \mathrm{SAH}$ patients before and after treatment. We found $41.6 \%$ on-admission ECG changes and 5\% echocardiographic abnormalities that were dropped to $6.7 \%$ and $1.7 \%$ after treatment, respectively. Abnormal ECG was a good predictor of inpatient mortality, but abnormal echocardiography was not.

\section{Competing Interest}

The authors declare that there is no competing interest.

\section{Grant Support}

All expenses of the present study were provided by a grant from Iran University of Medical Sciences.

\section{References}

1. Suarez JI, Tarr RW, Selman WR. Aneurysmal subarachnoid hemorrhage. N Engl J Med. 2006;354(4):387-396.

2. Johnston SC, Selvin S, Gress DR. The burden, trends, and demographics of mortality from subarachnoid hemorrhage. Neurology. 1998;50(5):1413-1418.

3. Kotila M. Declining incidence and mortality of stroke? Stroke. 1984;15(2):255-259.

4. Longstreth WT, Jr., Nelson LM, Koepsell TD, van Belle G. Clinical course of spontaneous subarachnoid hemorrhage: a population-based study in King County, Washington. Neurology. 1993;43(4):712-718.

5. McGirt MJ, Mavropoulos JC, McGirt LY, Alexander MJ, Friedman AH, Laskowitz DT, Lynch JR. Leukocytosis as an independent risk factor for cerebral vasospasm following aneurysmal subarachnoid hemorrhage. J Neurosurg. 2003;98(6):1222-1226.

6. Parkinson D, Stephensen S. Leukocytosis and subarachnoid hemorrhage. Surg Neurol. 1984;21(2):132-134.

7. Nakagawa I, Kurokawa S, Nakase H. Hyponatremia is predictable in patients with aneurysmal subarachnoid hemorrhage--clinical significance of serum atrial natriuretic peptide. Acta Neurochir (Wien). 2010;152(12):21472152.

8. Qureshi AI, Suri MF, Sung GY, Straw RN, Yahia AM, Saad M, Guterman LR, et al. Prognostic significance of hypernatremia and hyponatremia among patients with aneurysmal subarachnoid hemorrhage. Neurosurgery. 2002;50(4):749-755; discussion 755-746.

9. Kim YW, Neal D, Hoh BL. Risk factors, incidence, and effect of cardiac failure and myocardial infarction in aneurysmal subarachnoid hemorrhage patients. Neurosurgery. 2013;73(3):450-457; quiz 457. 
10. Ahmadian A, Mizzi A, Banasiak M, Downes K, Camporesi EM, Thompson Sullebarger J, Vasan R, et al. Cardiac manifestations of subarachnoid hemorrhage. Heart Lung Vessel. 2013;5(3):168-178.

11. Jeong YS, Kim HD. Clinically significant cardiac arrhythmia in patients with aneurysmal subarachnoid hemorrhage. J Cerebrovasc Endovasc Neurosurg. 2012;14(2):90-94.

12. Byer E, Ashman R, Toth LA. Electrocardiograms with large, upright $\mathrm{T}$ waves and long Q-T intervals. Am Heart J. 1947;33(6):796-806.

13. Di Pasquale G, Pinelli G, Andreoli A, Manini G, Grazi P, Tognetti F. Holter detection of cardiac arrhythmias in intracranial subarachnoid hemorrhage. Am J Cardiol. 1987;59(6):596-600.

14. Kreus KE, Kemila SJ, Takala JK. Electrocardiographic changes in cerebrovascular accidents. Acta Med Scand. 1969;185(4):327-334.

15. Solenski NJ, Haley EC, Jr., Kassell NF, Kongable G, Germanson T, Truskowski L, Torner JC. Medical complications of aneurysmal subarachnoid hemorrhage: a report of the multicenter, cooperative aneurysm study. Participants of the Multicenter Cooperative Aneurysm Study. Crit Care Med. 1995;23(6):1007-1017.

16. Burch GE, Meyers R, Abildskov JA. A new electrocardiographic pattern observed in cerebrovascular accidents. Circulation. 1954;9(5):719-723.

17. Shuster S. The electrocardiogram in subarachnoid haemorrhage. Br Heart J. 1960;22:316-320.

18. Harries AD. Subarachnoid haemorrhage and the electrocardiogram - a review. Postgrad Med J. 1981;57(667):294296.

19. Sakr YL, Lim N, Amaral AC, Ghosn I, Carvalho FB, Renard M, Vincent JL. Relation of ECG changes to neurological outcome in patients with aneurysmal subarachnoid hemorrhage. Int J Cardiol. 2004;96(3):369-373.

20. Cropp GJ, Manning GW. Electrocardiographic changes simulating myocardial ischemia and infarction associated with spontaneous intracranial hemorrhage. Circulation. 1960;22:25-38.

21. Banki NM, Kopelnik A, Dae MW, Miss J, Tung P, Lawton MT, Drew BJ, et al. Acute neurocardiogenic injury after subarachnoid hemorrhage. Circulation. 2005;112(21):3314-3319.

22. Cruickshank JM, Dwyer GN. Proceedings: Electrocardiographic changes in subarachnoid haemorrhage: role of catecholamines and effects of beta-blockade. Br Heart J. 1974;36(4):395.

23. Ananthachari MD, Anto CD. A study of ECG changes in 20 cases of subarachnoid haemorrhage. Indian Heart J. 1967;19(2):105-113.

24. Davies KR, Gelb AW, Manninen PH, Boughner DR, Bisnaire D. Cardiac function in aneurysmal subarach- noid haemorrhage: a study of electrocardiographic and echocardiographic abnormalities. $\mathrm{Br} \mathrm{J}$ Anaesth. 1991;67(1):58-63.

25. van den Bergh WM, Algra A, Rinkel GJ. Electrocardiographic abnormalities and serum magnesium in patients with subarachnoid hemorrhage. Stroke. 2004;35(3):644648.

26. Samuels MA. Neurogenic heart disease: a unifying hypothesis. Am J Cardiol. 1987;60(18):15J-19J.

27. Beattie JR, Cohen DJ, Manning WJ, Douglas PS. Role of routine transthoracic echocardiography in evaluation and management of stroke. J Intern Med. 1998;243(4):281291.

28. Mayer SA, LiMandri G, Sherman D, Lennihan L, Fink ME, Solomon RA, DiTullio M, et al. Electrocardiographic markers of abnormal left ventricular wall motion in acute subarachnoid hemorrhage. J Neurosurg. 1995;83(5):889896.

29. Mayer SA, Lin J, Homma S, Solomon RA, Lennihan L, Sherman D, Fink ME, et al. Myocardial injury and left ventricular performance after subarachnoid hemorrhage. Stroke. 1999;30(4):780-786.

30. Papanikolaou J, Makris D, Saranteas T, Karakitsos D, Zintzaras E, Karabinis A, Kostopanagiotou G, et al. New insights into weaning from mechanical ventilation: left ventricular diastolic dysfunction is a key player. Intensive Care Med. 2011.

31. Mayer SA, Fink ME, Homma S, Sherman D, LiMandri G, Lennihan L, Solomon RA, et al. Cardiac injury associated with neurogenic pulmonary edema following subarachnoid hemorrhage. Neurology. 1994;44(5):815-820.

32. Doshi R, Neil-Dwyer G. A clinicopathological study of patients following a subarachnoid hemorrhage. J Neurosurg. 1980;52(3):295-301.

33. Zaroff JG, Rordorf GA, Ogilvy CS, Picard MH. Regional patterns of left ventricular systolic dysfunction after subarachnoid hemorrhage: evidence for neurally mediated cardiac injury. J Am Soc Echocardiogr. 2000;13(8):774779.

34. Coghlan LA, Hindman BJ, Bayman EO, Banki NM, Gelb AW, Todd MM, Zaroff JG. Independent associations between electrocardiographic abnormalities and outcomes in patients with aneurysmal subarachnoid hemorrhage: findings from the intraoperative hypothermia aneurysm surgery trial. Stroke. 2009;40(2):412-418.

35. Crago EA, Kerr ME, Kong Y, Baldisseri M, Horowitz M, Yonas H, Kassam A. The impact of cardiac complications on outcome in the SAH population. Acta Neurol Scand. 2004;110(4):248-253.

36. Yarlagadda S, Rajendran P, Miss JC, Banki NM, Kopelnik $\mathrm{A}, \mathrm{Wu} \mathrm{AH}, \mathrm{Ko} \mathrm{N}$, et al. Cardiovascular predictors of in-patient mortality after subarachnoid hemorrhage. Neurocrit Care. 2006;5(2):102-107. 